\title{
MODELO DINÂMICO POR BALANÇO DE MASSA E ENERGIA EM CONVERSOR LD*
}

\author{
Alexandre Furtado Ferreira ${ }^{1}$ \\ Antônio Augusto de Resende Martins ${ }^{2}$ \\ Antônio Marcos Pereira ${ }^{3}$ \\ Fábio Oliveira da Silva ${ }^{4}$ \\ Flávio Ferreira ${ }^{5}$ \\ Rodrigo da Silva Magalhães ${ }^{6}$
}

\section{Resumo}

O presente trabalho descreve o desenvolvimento de um modelo matemático de conservação de massa e energia tendo como base a medição intermediária da sublança (Modelo Dinâmico). O modelo utiliza como dados de entrada informações sobre massa e composição da carga metálica, valores adicionados de fundentes na corrida e as informações obtidas com a medição intermediária aos $88 \%$ do sopro de oxigênio calculado pelo Modelo Estático. O algoritmo foi desenvolvido em C\# e está integrado na rede de Nível 2 da CSN. Os resultados de acerto simultâneo de temperatura e carbono final de sopro obtido pelo modelo dinâmico foram superiores ao Modelo Estatístico até então utilizado. Tal fato permitiu a redução do ressopro decorrentes de imprecisão do modelo e ganho em produtividade.

Palavras-chave: Modelo dinâmico; Balanço de massa e energia; Conversor LD.

\section{DYNAMIC MODEL FOR MASS AND ENERGY BALANCE IN LD CONVERTER Abstract}

This paper describes the development of a mathematical model of mass and energy conservation, it based on the intermediate sublança measurement (Dynamic Model). The model uses, as input, information about weight and composition of metallic charge, weight of the lime and measurements realized by sublance when there is $88 \%$ of de heat oxygen blowing, that was calculated earlier through the static model. The new mathematical model is integrated into CSN Level 2 network. The results of simultaneous adjustment of temperature and final carbon content is obtained by the new Dynamic Model was higher than the statistical model used usually. This fact allowed the reduction of new oxygen blowing due to inaccuracy of the statistical model and gain in steel productivity.

Keywords: Dynamic model; Mass and energy balance; LD converter.

1 Metalurgia, Doutorado, Professor, Departamento de Engenharia Mecânica, UFF, Volta Redonda, RJ, Brasil.

2 Metalurgia, Engenheiro, Coordenador de Projetos Especiais, Área Técnica, CSN, Volta Redonda, RJ, Brasil.

3 Matemática, Matemático, Analista Operacional, Gerência de Aciaria, CSN, Volta Redonda, RJ, Brasil.

4 Análise de Sistema, Analista, Analista Especialista, Gerência de Tecnologia, CSN, Volta Redonda, RJ, Brasil.

5 Metalurgia, Doutorado, Professor, Departamento de Engenharia de Produção, UFF, Volta Redonda, RJ, Brasil.

6 Metalurgia, Engenheiro, Engenheiro Especialista, Área Técnica, CSN, Volta Redonda, RJ, Brasil. 


\section{INTRODUÇÃO}

O desafio em aliar qualidade e produtividade é um ponto fundamental na siderurgia mundial. Tendência moderna em controle de processo os modelamentos matemáticos são ferramentas efetivas. Nas aciarias estes softwares de supervisão estão instalados em estações de operação que interagem com os CLP's através de redes de comunicação industriais. Habitualmente classificado como nível 2 este método de operação tem sido aplicada com maior intensidade nas últimas décadas impactando diretamente no desempenho dos processos [1].

Dentro deste contexto é proposto o desenvolvimento e avaliação de um modelo matemático de conservação de massa e energia tendo como parte dos parâmetros de entrada os valores medidos pela sublança. O modelo utiliza informações de massa e composição da carga metálica e adições realizadas durante o sopro de oxigênio bem como os dados de percentual de carbono e temperatura medidos pela sublança na $3^{\circ}$ etapa do sopro. Como resultado é calculado a real necessidade de volume de oxigênio e agente endotérmico que precisam ser utilizados para que os valores objetivados de percentual de carbono e temperatura sejam alcançados. Dessa forma é possível a redução da ocorrência de ressopro ou resfriamento da corrida após término do sopro de oxigênio e, consequentemente, a redução do consumo de desoxidante, aumento do rendimento metálico e ganho de produtividade. A tabela 1 mostra de forma quantitativa o impacto do ressopro nas variáveis operacionais:

Tabela 1 - Impacto do ressopro no processo de refino primário

\begin{tabular}{|c|c|}
\hline \multicolumn{2}{|c|}{ Corrida ressoprada } \\
\hline Variável & Impacto médio/corrida \\
\hline ppm Oxigênio & aumento de $340 \mathrm{ppm}$ \\
\hline Fe na escória & aumento de $1.9 \mathrm{t}$ \\
\hline Tempo FS-IV & aumento de $4 \mathrm{~min}$ \\
\hline Consumo de Alumínio & aumento de $48 \mathrm{Kg}$ \\
\hline
\end{tabular}

\section{MATERIAIS E MÉTODOS}

$\mathrm{Na}$ siderurgia todos os modelos que utilizam como base de entrada os dados da medição realizada pela sublança durante o sopro são denominados de dinâmico. Seja qual for à metodologia adotada o objetivo é calcular a necessidade de oxigênio e agente endotérmico que ainda precisam ser adicionados na corrida após a medição com a sublança. Com isso é possível corrigir o "caminho" traçado pelo Modelo Estático para que a faixa definida de percentual de carbono e temperatura seja alcançada ao término do sopro (figura 1). 


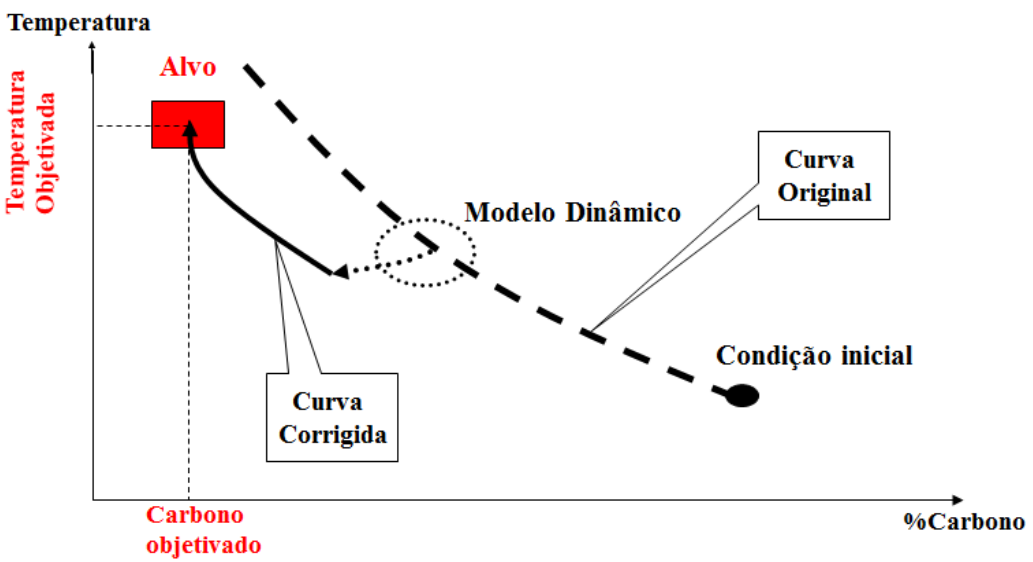

Figura 1 - Curva do Modelo Estático corrigida pelo Modelo Dinâmico

A precisão do Modelo Dinâmico é superior a do Modelo Estático uma vez que restringe o número de incógnitas e variáveis de entrada. Além disso, há uma maior precisão dos valores coletados pelo fato de que no momento de seu cálculo toda adição de fundentes, escorificantes, agente endotérmico ou exotérmico já foram realizadas e o banho se encontra homogêneo.

O trabalho desenvolvido tem como base um balanço de massa e energia entre 0 momento da medição intermediária do banho e o término do sopro de oxigênio. Para que os cálculos possam ser efetivados é necessário utilizar dados sobre peso e composição da carga metálica, adição de fundentes, valores medidos pela sublança, valores objetivados de carbono e temperatura ao final do sopro, peso e composição de fundentes adicionados e constantes termodinâmicas. Além disso, é necessário o desenvolvimento de equações empíricas para estimar algumas variáveis importantes para o modelamento.

\subsection{BALANÇO DE MASSA}

Consiste em determinar as massas de cada componente no inicio e final do processo que está sendo considerado. Dessa forma é possível calcular a quantidade de oxigênio necessária para gerar a oxidação dos elementos presentes no aço e a quantidade de oxigênio que ficará dissolvida no banho. Desse total deverá ser subtraído o aporte de oxigênio proveniente do minério de ferro que venha a ser adicionado no final do processo. Uma vez que na terceira etapa do sopro não há mais silício presente no banho não ocorrerá mais formação de $\mathrm{SiO}_{2}$. Para simplificação foi considerado que a massa de enxofre presente no banho não sofre alteração. Essa hipótese é bastante razoável uma vez que a remoção do enxofre em ambiente oxidante, como é o caso do conversor, não é eficiente. A equação 1 retrata as massas envolvidas no cálculo:

(1) Massa oxigênio necessária após sl1

= Oxigênio no $\mathrm{FeO}$ após sl1 + Oxigênio no CO após sl1

+ Oxigênio no $\mathrm{CO}_{2}$ após sl1 + Oxigênio no MnO após sl1

+ Oxigênio no $\mathrm{P}_{2} \mathrm{O}_{5}$ após sl1 + Peso oxigênio dissolvido sl3

- Oxigênio minério adição sl1 
Onde:

Massa Oxigênio necessária após sl1 = massa de oxigênio necessária para oxidar os elementos após a medição intermediária e para incorporar no banho $(\mathrm{kg})$

Oxigênio no FeO após sl1 = massa de oxigênio necessária para oxidar o ferro após medição intermediária $(\mathrm{kg})$

Oxigênio no $\mathrm{CO}$ após sl1 = massa de oxigênio necessária para formar gás CO após medição intermediária $(\mathrm{kg})$

Oxigênio no $\mathrm{CO}_{2}$ após sl1 = massa de oxigênio necessária para formar gás $\mathrm{CO}_{2}$ após medição intermediária $(\mathrm{kg})$

Oxigênio no $\mathrm{MnO}$ após sl1 = massa de oxigênio necessária para formar $\mathrm{MnO}$ após medição intermediária $(\mathrm{kg})$

Oxigênio no $\mathrm{P}_{2} \mathrm{O}_{5}$ após sl1 = massa de oxigênio necessária para formar $\mathrm{P}_{2} \mathrm{O}_{5}$ após medição intermediária $(\mathrm{kg})$

Peso Oxigênio Dissolvido $\mathrm{s} \mid 3=$ peso estimado de oxigênio dissolvido no aço ao final de sopro $(\mathrm{kg})$

Oxigênio no Minério Calc após sl1 = massa de oxigênio presente no Minério de Ferro calculado para adição após medição intermediária $(\mathrm{kg})$

\subsection{BALANÇO DE ENERGIA}

Pelo princípio de conservação de energia pode-se afirmar que o total de entalpia que entra no sistema é igual ao total de entalpia que saí do sistema menos as perdas. Tal principio está descrito na equação 2:

(2) $\Delta$ H entrada $=\Delta$ Saída $+\Delta$ Perdas

Onde:

$\Delta \mathrm{H}$ entrada $=$ entalpia total fornecida ao sistema após a medição intermediária até o final de sopro (kcal)

$\Delta \mathrm{H}$ Saída= entalpia total consumida pelo sistema entre a medição intermediária e a medição final de sopro (kcal)

$\Delta \mathrm{H}$ Perdas $=$ entalpia total perdida pelo sistema entre a medição intermediária e a medição final de sopro (kcal)

Para calcular o modelo de conservação de energia todas as massas são conhecidas com exceção do minério de ferro. A quantidade calculada de minério de ferro será a necessária para igualar a entalpia de entrada com a entalpia de saída do sistema mais as perdas. 


\subsubsection{ENTRADA DE CALOR NO SISTEMA}

A entrada total de calor é dada pela equação 3:

(3) $\Delta$ H entrada

$=\Delta H$ escória entrada $+\Delta$ Haço entrada + Entalpia Reação
+ Entalpia de Decomposição + Entalpia de Dissolução

Onde:

$\Delta \mathrm{H}$ entrada $=$ entalpia total fornecida ao sistema após a medição intermediária até o final de sopro (kcal)

$\Delta \mathrm{H}$ escória entrada $=$ entalpia fornecida pelos componentes da escória no momento da medição intermediária (kcal)

$\Delta \mathrm{H}$ aço entrada = entalpia fornecida pelos componentes do aço no momento da medição intermediária (kcal)

$\Delta \mathrm{H}$ entalpia reação = entalpia fornecida pela formação dos óxidos após medição intermediária (kcal)

Entalpia de decomposição= entalpia de decomposição dos elementos presentes no aço líquido no momento da medição intermediária (kcal)

Entalpia de dissolução= entalpia de dissolução dos elementos presentes no aço líquido no final de sopro (kcal)

Para que as equações de mudança de entalpia devido à temperatura possam ser aplicadas, é necessário decompor os elementos dissolvidos no aço no momento da medição intermediária do sopro. Ao final do processo faz-se a operação contrária, ou seja, verificam-se os elementos presentes e calcula-se sua dissolução no aço líquido. A dissolução do carbono no aço é positiva, ou seja, é uma reação endotérmica. Já a dissolução do fósforo e oxigênio é uma reação exotérmica. $O$ resultado é oposto ao se analisar a entalpia de decomposição. A entalpia de dissolução/decomposição do manganês é considerada nula e por isso não gera e não consome energia. Ao se analisar a entalpia para dissolução e decomposição do carbono, fósforo e oxigênio chega-se a conclusão, para os valores aqui praticados, que ambos os casos possuem balanço de entalpia negativo, ou seja, geram calor para o sistema e por esse motivo ambas foram consideradas entrada de calor. $O$ ferro não necessita ser dissolvido uma vez que é fundamentalmente a matriz do sistema considerado. As entalpias de solução, decomposição, calor de formação e de aquecimento dos elementos/óxidos tem como base os dados do BOF Steelmaking $[2,3]$. 


\subsubsection{SAÍDA DE CALOR NO SISTEMA}

A saída total de calor é dada pela equação 4:

(4) $\Delta H$ saída $=\Delta H$ escória saída $+\Delta H$ aço saída $+\Delta H$ gases saída $+\Delta H$ minério $+\Delta H$ perdas

Onde:

$\Delta \mathrm{H}$ saída= entalpia total consumida pelo sistema entre a medição intermediária e a medição final de sopro (kcal)

$\Delta \mathrm{H}$ escória saída = variação de entalpia dos componentes da escória de $298 \mathrm{~K}$ até a temperatura objetivada final de sopro em Kelvin (kcal)

$\Delta \mathrm{H}$ aço saída= entalpia para aquecer a composição do aço final de sopro de $298 \mathrm{~K}$ a temperatura objetivada final de sopro (kcal)

$\Delta \mathrm{H}$ gases saída= entalpia de aquecimento dos gases $\mathrm{CO}$ e $\mathrm{CO}_{2}$ de $298 \mathrm{~K}$ até a temperatura de referência de aquecimento dos gases

$\Delta \mathrm{H}$ minério= entalpia consumida pela adição do minério após medição intermediária (kcal)

$\Delta \mathrm{H}$ perdas= estimativa de perda de calor do sistema após medição intermediária (kcal)

\subsubsection{PERDA TÉRMICA}

A perda térmica do sistema é considerada como um determinado percentual da entalpia de entrada. Seu cálculo é considerado em relação ao tempo de conversor parado (equação 5).

(5) $\Delta H$ perdas $=$ Constante Tempo de Conversor Parado $* \Delta H$ entrada

Onde:

$\Delta \mathrm{H}$ perdas $=$ entalpia total consumida pelo processo após a medição intermediária (kcal)

Constante Tempo de Conversor parado = percentual de perda relativo ao tempo de conversor parado

$\Delta \mathrm{H}$ entrada= entalpia total fornecida ao sistema após a medição intermediária até o final de sopro (kcal)

A "Constante Tempo de Conversor parado" é determinada experimentalmente de acordo com os dados reais do processo. Após o equacionamento dos balanços de massa e energia o modelo dinâmico obtém como resposta o volume de oxigênio a 
ser soprado e o peso de agente endotérmico que necessita ser adicionado na corrida para que os valores objetivados de carbono e temperatura (acerto simultâneo) sejam alcançados no final do processo de refino primário.

\subsection{VARIÁVEIS QUE INTERFEREM NO CÁLCULO DO MODELO}

No momento da medição intermediária do banho pela sublança há três fatores importantes que interferem diretamente no cálculo do Modelo Dinâmico e, consequentemente, no acerto simultâneo. $O 1^{\circ}$ fator preponderante é a falta de reserva térmica no momento da medição intermediária. Quando isso ocorre o Modelo Dinâmico calcula um excesso de oxigênio visando uma maior oxidação de carbono e ferro resultando em uma maior geração de calor para o sistema. Dessa forma há uma grande possibilidade de o teor de carbono obtido ao final do sopro ser inferior ao mínimo da faixa de acerto. $\mathrm{O} 2^{\circ}$ fator é justamente o oposto do discutido anteriormente. Se no momento da medição intermediária a reserva térmica do sistema estiver demasiadamente elevada uma grande quantidade de agente endotérmico será calculada pelo modelo e poderá não haver tempo hábil para que ocorra sua total dissolução no banho. O $3^{0}$ fator diz respeito à precisão do teor de carbono no banho na medição intermediária. Como não há tempo hábil para aguardar a análise laboratorial se faz necessário uma estimativa do percentual de carbono através da temperatura líquidus do banho (figura 2). Como o percentual de carbono é uma das variáveis de entrada com maior peso no cálculo do modelo, uma estimativa imprecisa certamente impactará no resultado do modelo.

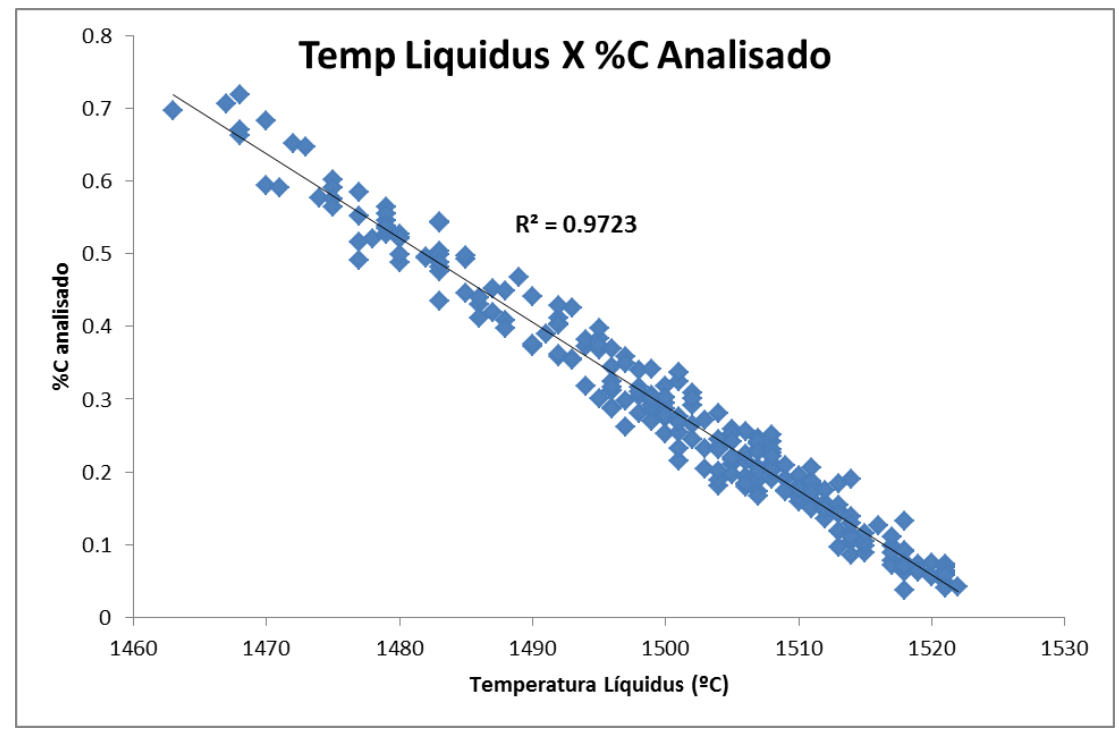

Figura 2 - \%Carbono x Temperatura Líquidus

\section{RESULTADOS E DISCUSSÃO}

O histograma da figura 3 mostra a diferença entre o valor de oxigênio calculado pelo modelo dinâmico e o valor efetivamente utilizado no processo. O operador tem a autonomia de soprar até $200 \mathrm{Nm}^{3}$ de oxigênio a mais ou a menos do valor calculado pelo modelo dinâmico. É observado que o cálculo de oxigênio do modelo dinâmico tem sido seguido dentro das tolerâncias estabelecidas. 


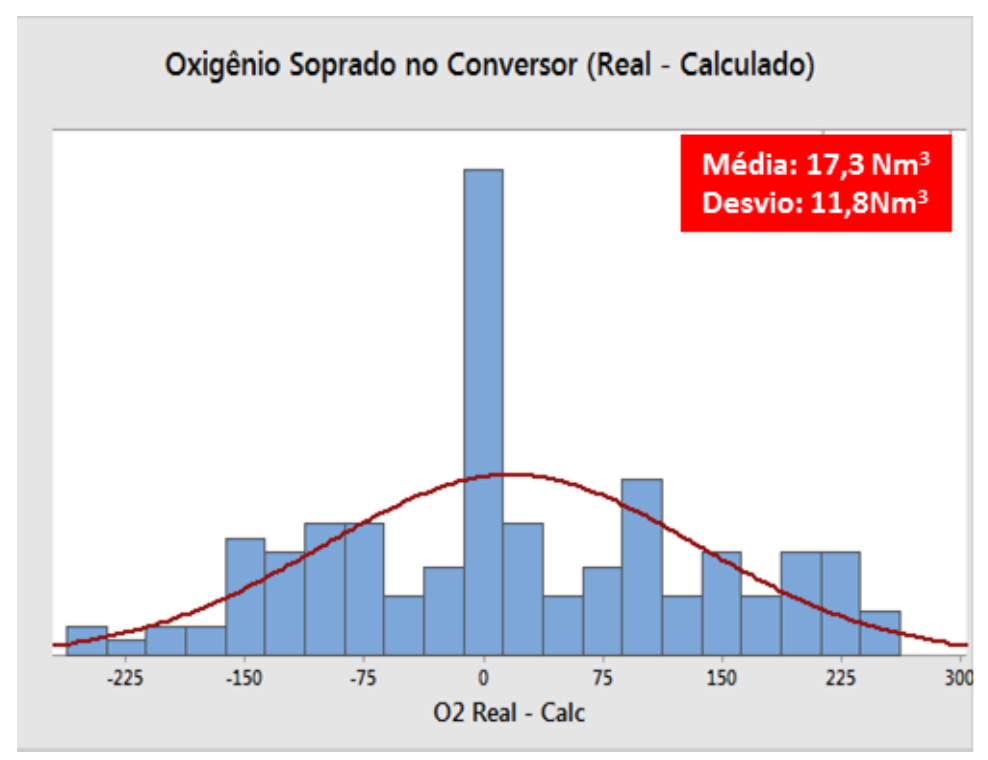

Figura 3 - Diferença entre o oxigênio real e o calculado pelo modelo dinâmico

A figura 4 mostra o histograma da diferença entre o valor de minério calculado pelo modelo dinâmico e o efetivamente adicionado na corrida. $O$ operador tem a autonomia de adicionar $500 \mathrm{~kg}$ a mais ou a menos do que o valor calculado pelo modelo. Em média adiciona-se cerca de $70 \mathrm{~kg}$ a mais de minério em relação ao calculado. Essa diferença está relacionada à precisão do sistema de adição de minério.

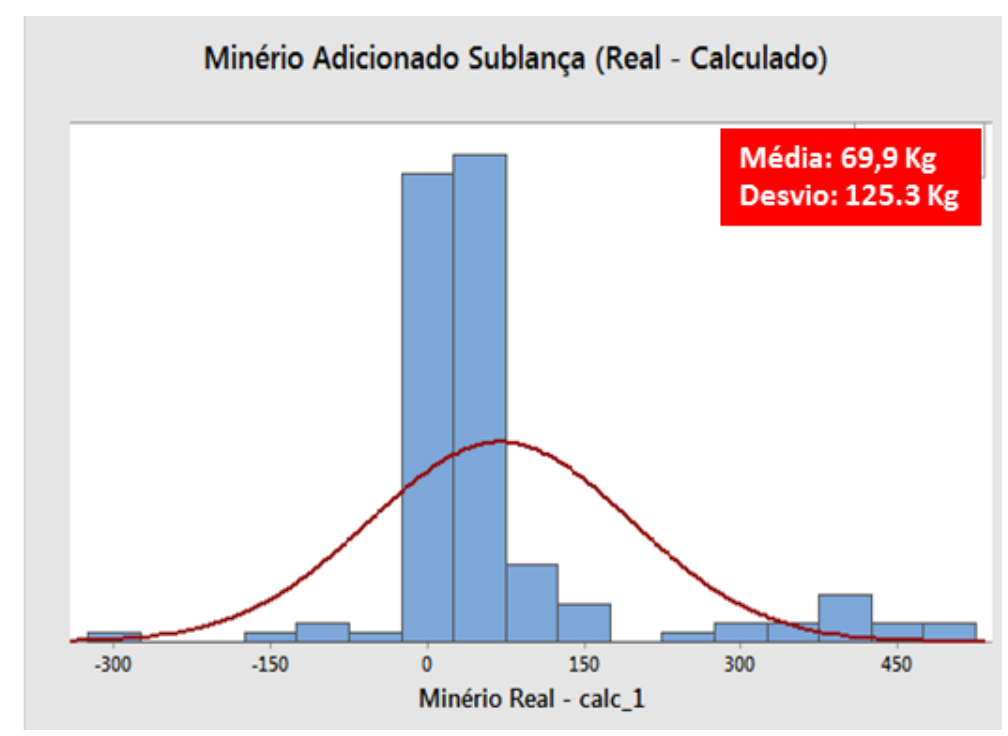

Figura 4 - Diferença entre o minério adicionado e o calculado pelo modelo dinâmico

A figura 5 descreve o percentual de acerto simultâneo de carbono e temperatura entre o modelo dinâmico estatístico e o modelo dinâmico por balanço de massa e energia. 


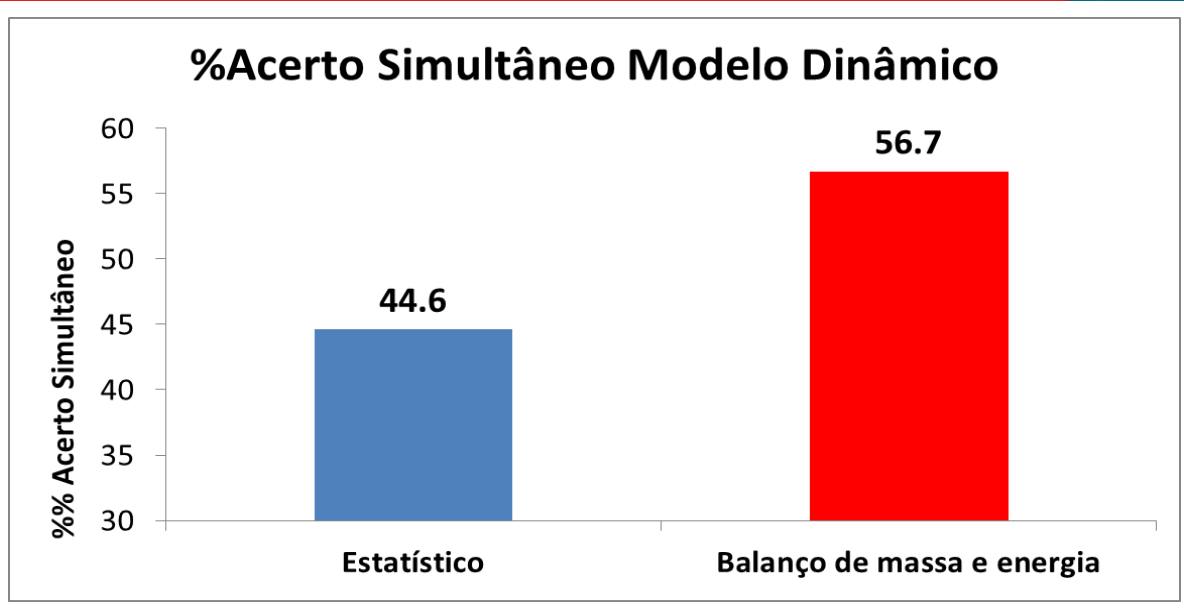

Figura 5 - Comparação do \%acerto simultâneo do modelo dinâmico estatístico e o por balanço de massa e energia

A figura 6 mostra a influência no acerto simultâneo da condição da reserva térmica da corrida e da precisão da estimativa do teor de carbono no momento da medição intermediária.

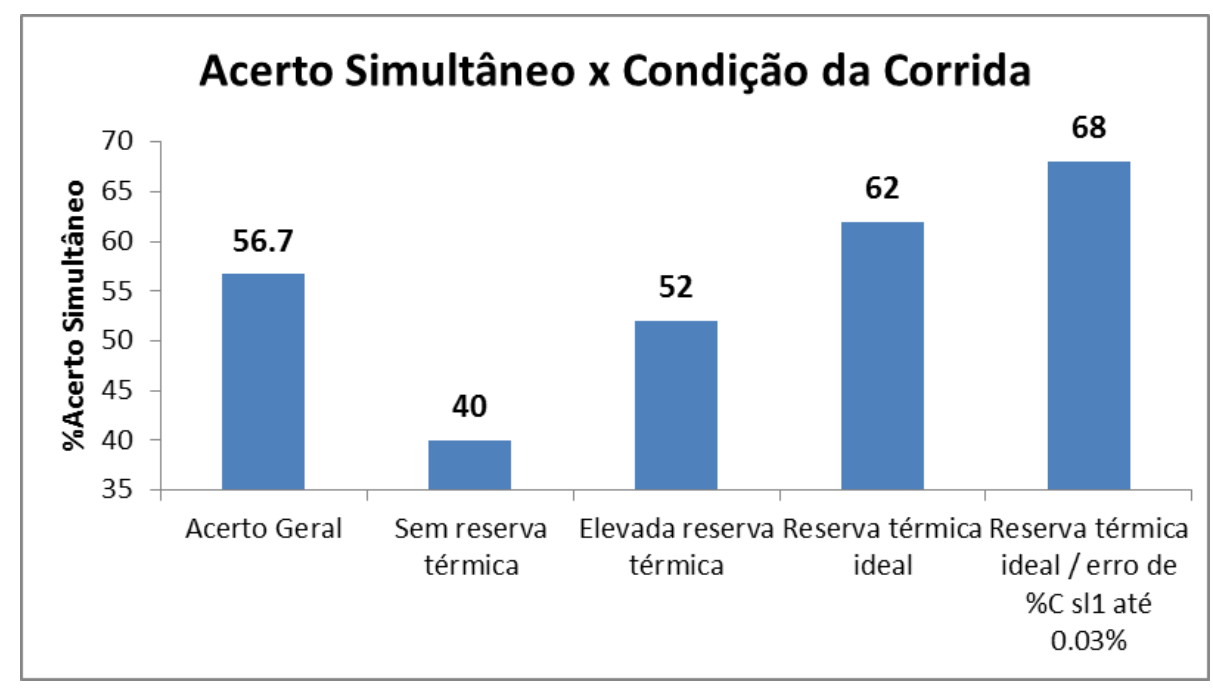

Figura 6 - Acerto Simultâneo x Condição da Corrida

O ressopro é considerado uma anomalia e como toda anomalia há uma causa fundamental. A figura 7 mostra a participação da imprecisão do modelo na ocorrência do ressopro, ou seja, do total de corridas ressopradas qual o percentual foi devido a erro de cálculo do modelo. 


\section{\%Ressopro devido ao Modelo Dinâmico}

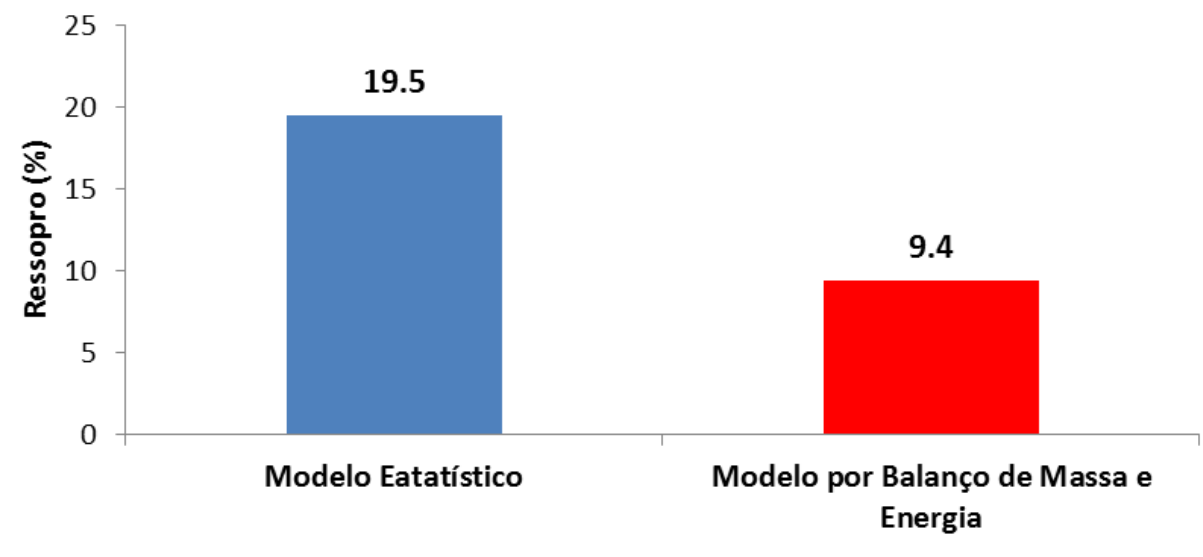

Figura 7 - Participação da imprecisão do modelo na ocorrência do ressopro

\section{CONCLUSÃO}

O modelo dinâmico, com base no principio de conservação de massa e energia, representa uma abordagem diferenciada da que é tradicionalmente feita pelas usinas siderúrgicas. Por esse motivo seu desenvolvimento e aplicação torna-se um interessante desafio ainda mais devido ao fato de ser difícil prever e quantificar o comportamento de uma série de variáveis nos instantes finais do sopro. Os resultados iniciais são favoráveis quando comparado ao Modelo Estatístico por regressão linear múltipla utilizado anteriormente.

\section{REFERÊNCIAS}

1 Magalhães, R.S; Martins, A. A. R; Barbosa, W. F. Modelagem Matemática do Refino em Forno Panela na CSN. XLII Seminário de Aciaria - Internacional, da Associação Brasileira de Metalurgia e Materiais, 15 a 18 de maio de 2011, Salvador, Ba, Brasil;

2 BOF Steelmaking, ISS-AIME,1982, Vol II, Table 13-3 - Enthalpy Changes Due to Temperature

3 BOF Steelmaking, ISS-AIME,1982, Vol II, Table 13-4 - Enthalpies of Reaction 